\title{
Approach of a Tanker Aircraft to the Remote- Controlled Drogue of the Air-to-Air Refueling System
}

\author{
M.K. Filyashkin \\ National Aviation University \\ Kyiv, Ukraine \\ filnik@ukr.net
}

\begin{abstract}
Here are considered benefits of airlift using an airto-air refueling civil aircraft. Such technologies provide in particular for regional aircraft their main advantages at long flights. There are proposed variants of automation of approach of refueling devices using a predictive model.
\end{abstract}

Keywords-efueling; probe of refueling; drogue; refueled aircraft; tanker aircraft; control laws; remote-controlled drogue

\section{INTRODUCTION}

To optimize air transportation, it is not only necessary to choose the type of vehicle, but also to ensure the efficiency of its uses with the apply of modern transport technologies. Improving economic efficiency of air transport services can be accomplished through the use for regional aircraft technology of air-to-air refueling (AAR), which can provide the exploitation of these aircraft on long-haul non-stop flights instead of expensive long-haul aircraft [1].

A striking example of regional jets, for which can be applied, these technologies are modern jets: An-158 (passenger variant) and An-178 (transport aircraft) of the company Antonov. Comparative analysis of the technical characteristics of the aircraft An -158, characteristics of the aircraft of mediumrange Boeing 737-900 and the long-range aircraft A330-300 of the company Airbus shows that short-haul (SHA) and medium-haul aircraft (MHA) can well solve the problem of transportation of passengers on long international lines. In this case the characteristics of navigation equipment of the aircrafts approximately the same and a flight comfort on SHA and on MHA is not worse than on the elite long-haul mainline jets (LHA). In addition, service for a smaller number of passengers could be better and it will cost less resource for the airline. The problem of LHA is also the fullness of load flights, which is low for the long international lines.

The AAR technology can significantly reduce the fuel consumption, will allow several times to increase the commercial aircraft load and reduce the emissions of $\mathrm{CO}^{2}$, as well as reduce the cost of the flight fleet.

In addition, the implementation of direct long-haul flights using regional aircrafts proportion of flights with a stopover in the airline hub is reduced, because passengers can be delivered directly to the destination without change of flights in international airports.
All this emphasizes the urgency of developing AAR technologies for civil aircraft. The development of this idea is currently engaged in leading aviation research organizations in Europe in the framework of "Recreate" project [2], as well as scientific institutions of Russia and Israel. Several US companies, including Northrop Grumman, are also developing automatic air-fueling systems.

\section{Problem Statement}

Analysis of existing refueling systems shows that the most common and relatively simple system is the "probe-and-drogue system". Therefore this method is taken as the basis for the development of the automated system of AAR for civil aircraft.

But in order to reduce the requirements for pilots of civil airliners in the article is proposed for the "probe-and-drogue" system use the method of "inverse" AAR (Fig. 1).

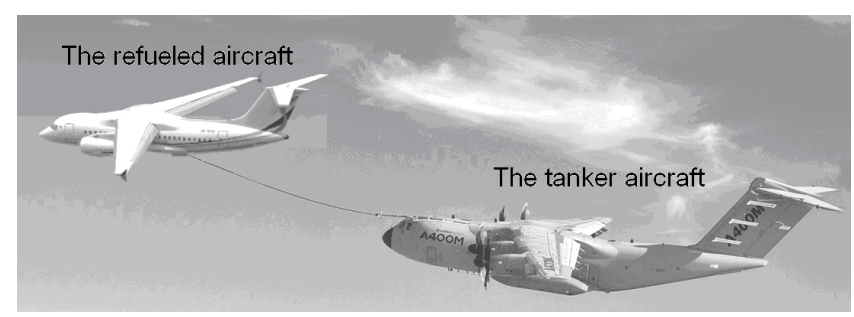

Fig.1. Method of "inverse" air-to-air refueling.

This method requires no special training pilots of the the refueled aircraft. The entire alignment process of rapprochement and contact performs a trained crew of the tanker aircraft, which is approaching to the commercial aircraft behind and performing the maneuvers, connects its own fueling system with the extended drogue.

Special modifications of civil aircraft, as an aircraft that refuel in the air, is not required, if using a unified outboard unit of refueling that is suspended under the fuselage of the refueled aircraft.

At the same time it's necessary to automate not only the control of the aircraft, as well as the control of a drogue of AAR, equipping the drogue aerodynamic control surfaces and thereby turning it into the remote-controlled UAV, which the crew of tanker will be able to direct on its probe of a refueling system. 
Additionally refueling process can be automated to reduce the load on the tanker aircraft crew.

In the process of contacting (approach the probe of the tanker aircraft to the drogue beginning from a distance of $15-10 \mathrm{~m})$, it is necessary to provide precise control over the height and lateral deviation, as well as the speed of approach. The presence of two pilots on the airplane-tanker allows dividing the control process.

In particular, the second pilot is entrusted with the task of directing and holding a drogue which turned into a remote controlled UAV to expand probe axis refueling system. In this case, the drogue control system can improve the process of directing and stabilizing the drogue in space, parrying the turbulent perturbations. The structure and research results of a control system of this type are given in [3]. The precision of keeping the coordinates of the drogue when using such a system is $0.2-0.3 \mathrm{~m}$.

When using the technology of "inverse" AAR, the refueled aircraft let out a "dry" hose-drogue that is unfilled with fuel and enable autopilot in the mode of automatic stabilization of altitude and flight speed. By supplying compressed air into the hose, it is possible to use its energy to control the drogue. For this purpose, the adjustable nozzles 2 are located pairwise on the diametrically opposite sides of the casing of the refueling drogue and are connected by slit channels with a hose. The release of compressed air through the nozzles alters the flow of air jets along the aerodynamic profile of the drogue and thus changes the distribution of the aerodynamic forces acting on the drogue, that is allows you to control the movement of the drogue in the airflow (Fig. 2).

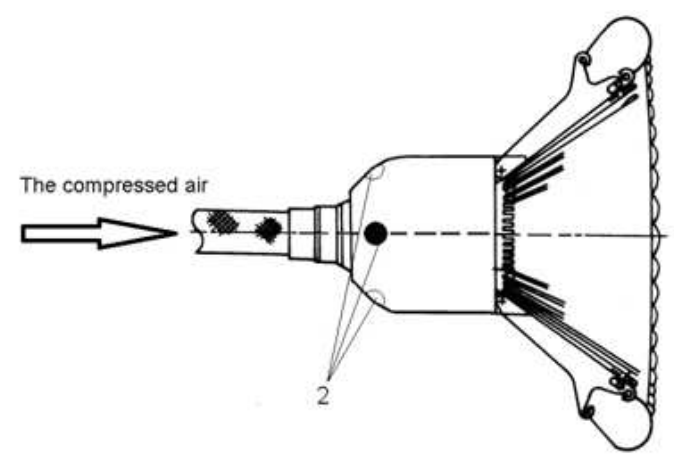

Fig.2. Control drogue nozzles.

The first pilot solves the following tasks: meeting with the refueled aircraft, aligning the tanker aircraft and the refueled aircraft and exiting to the height of the average drogue position. The most important operation of the AAR is the approach and docking of the fuel probe with the drogue, which starts at a distance of $15-10 \mathrm{~m}$.

Accuracy of retention the approach speed should exceed the speed of the refueled aircraft by 1-2 m/s. At a lower approach speed, the cone may not work, as a result of which the contact time increases, and the tanker aircraft sways in the turbulent flow. At a higher approach speed, a strong impact on the drogue takes place at the moment of contact, which leads to an oscillating motion of the hose (the "whip effect") and to the rocking of the drogue in the vertical plane. In this case, as a rule, there is destruction (bummer) of the fuel receiver or breakage of the hose.

Therefore, the successful docking of refueling devices during «inverse» AAR largely depends on the skill and physiological condition of the tanker crew. The creation of systems with a controlled drogue greatly simplifies the process of docking the refueling devices; however, the problem of maintaining an approach speed is not removed.

In the article, it is proposed to form a flexible approach trajectory based on the method of controlling the final state when docking the refueling devices. If the tanker aircraft leaves the primary trajectory, then realizing this control method, a new trajectory is formed from the new location of the tanker, leading the aircraft to the specified end point. A necessary element of such a control method is prediction of the tanker movement parameters. Therefore, the problem statement can be formulated as follows: for the process of approach of refueling devices, obtain an algorithm for controlling the tanker aircraft speed using a predictive model.

\section{PROBLEM SOLUTION}

The theory of control of dynamic objects using predictive models - (Model Predictive Control (MPC)) is one of the modern formalized approaches to the synthesis of control systems based on mathematical methods of optimization. The advantage of MPC, which determines its successful use in practice of creation of control systems, is the relative simplicity of the basic feedback generation scheme, combined with high adaptive properties.

The essence of MPC is the following scheme of controlling of dynamic objects based on the feedback principle:

1. Is considered a certain (relatively simple) mathematical model of the object, the initial conditions for which are its current parameters of motion. Assigning the program of control, the equations of this model are integrated, which gives a prediction of the motion of the object at some finite time interval (at the prediction horizon).

2. The optimization of program control is performed, the purpose of which is to approximate the adjustable variables of the predictive model to the corresponding parameters set on the prediction horizon. The optimization is carried out taking into account the whole complex of constraints imposed on the control and adjustable variables.

3. At the step of calculation, which constitutes a fixed small part of the prediction horizon, an optimal computed control is realized and the actual state of the object is measured at the end of this step.

4. The prediction horizon is moved one step further, and points $1 \ldots 3$ of this sequence of actions are repeated.

The above scheme can be combined with the preliminary identification of equations of model used for the prediction.

At the stage of docking approach with the use of predictive, the important point is to select an independent variable for predicting. Obviously, as an independent variable, one of 
the coordinates must be taken, the value of which determines the end of the docking approach. Such a coordinate when controlling the speed of the tanker aircraft, it is advisable to choose the distance between the refueling devices.

For the control object of a mathematical model, there is a system of nonlinear differential equations of the form:

$$
\dot{\mathbf{x}}+f(\mathbf{x}, \mathbf{u})=0, \quad \mathbf{x}(0)=\mathbf{x}_{0},
$$

where $\mathbf{x} \in \mathbf{E}^{n}$ is the state vector; $\mathbf{u} \in \mathbf{E}^{m}$ is the control vector; $t \in[0, \infty]$ is the time.

Simultaneously with the mathematical model (1) of the control object, is view a system of differential equations of the form:

$$
\dot{\overline{\mathbf{x}}}(\tau)+\bar{f}(\tau, \overline{\mathbf{x}}(\tau), \overline{\mathbf{u}}(\tau))=0,\left.\quad \overline{\mathbf{x}}\right|_{\tau=t}=\mathbf{x}(t),
$$

which is a predictive model in relation to the mathematical model (1) of the control object.

Obviously, the function $\bar{f}$ must have the same properties as the function $f$, and the vectors $\overline{\mathbf{x}}$ and $\overline{\mathbf{u}}$ take values from the admissible sets $\mathbf{x}$ and $\mathbf{u}$, respectively.

In addition, the function $\bar{f}$ is defined in such a way that for each admissible control $\overline{\mathbf{u}}(\tau) \equiv \mathbf{u}(\tau)$ vector functions $\mathbf{x}(\tau)$ and $\overline{\mathbf{x}}(\tau)$, satisfying systems (1) and (2), accordingly, are near to each other in the norm for each $\tau \in[t, \infty]$.

Model (1) in the process of functioning can change, and these changes are not a priori defined. At the same time, a fixed model of the type (2) is initialized at the instant $\tau=t$ by the current state of the real object, and due to its proximity to it for any variations of unaccounted factors, it allows approximate predicting its behavior. This can be done if we find an individual solution of the system (2) for a prescribed control over a certain interval of time $\tau \in[t, t+T]$, and the prediction will be more accurate, the smaller the value of $T$. Typically, the predictive model is chosen quite simply so that it can be integrated in real time and directly used in the control loop.

In the researches as a mathematical model of the control object, a mathematical model of the longitudinal motion of the aircraft was used:

$$
\begin{aligned}
& m \dot{V}=P \cos \alpha-X_{a}-m g \sin \Theta-m\left(\dot{w}_{x} \cos \Theta+\dot{w}_{y} \sin \Theta\right) ; \\
& m V \dot{\Theta}=P \sin \alpha+Y_{a}-m g \cos \Theta+m\left(\dot{w}_{x} \sin \Theta+\dot{w}_{y} \cos \Theta\right) ; \\
& J_{z} \dot{\omega}_{z}=M_{z} ; \\
& \dot{\vartheta}=\omega_{z} ; \\
& \alpha=\vartheta-\Theta+\alpha_{w} ; \\
& \dot{H}=V y=V \sin \left(\Theta+\alpha_{w}\right)+w_{y} ; \\
& \dot{D}=V \cos \left(\Theta+\alpha_{w}\right)+w_{x}, \\
& \dot{D}_{\mathrm{d}}=V_{\mathrm{rf}}+w_{x}, \\
& \Delta D=D-D_{\mathrm{d}},
\end{aligned}
$$

where $m$ is the aircraft weight; $M_{z}, J_{z}$ are moment of forces and moment of inertia of the aircraft relative to the transverse axis $z$; $P$ is the engine thrust; $X_{a}, Y_{a}$ is the force of drag and lift; $\vartheta$, $\Theta$ is the pitch and an angle of inclination of the trajectory; $\omega_{z}$ is the angular velocity with respect to the axis $z ; w_{x}, w_{y}$ are wind perturbation components; $\alpha, \alpha_{w}=\left(-w_{x} \sin \Theta\right.$ $\left.+w_{y} \cos \Theta\right) / V$ is the angle of attack and the increase in the angle of attack from the wind disturbance; $D$ is the tanker aircraft distance; $D_{\mathrm{d}}$ is the distance flown by the drogue; $V_{\mathrm{rf}}$ is the preset speed of refueling; $\Delta D$ is the distance between refueling devices. The aircraft is controlled with the elevator $\delta_{\mathrm{e}}$ by changing the moment $M_{z}=f\left(\delta_{\mathrm{e}}\right)$ and using the autothrottle system $P=f\left(\delta_{\mathrm{ECL}}\right)$; here $\delta_{\mathrm{ECL}}-$ the travel with the use of the autothrottle of the engine control levers (main control).

As a predictive mathematical model, a simplified model of the motion of the center of mass can be chosen. This can be a linear model of the change in the speed of flight $\mathrm{c}$ for a given flight regime - for regime of refueling, that in the operator form has the form

$$
\begin{aligned}
& \left(p+a_{x}^{V}\right) V(p)=a_{x}^{\delta_{p}} \delta_{\mathrm{ECL}}(p) \\
& p D=V(p) \\
& p D_{\mathrm{g}}=V_{\mathrm{rf}} \\
& \Delta D(p)=D(p)-D_{\mathrm{g}}(p)
\end{aligned}
$$

the coefficients $a_{x}^{V}, a_{x}^{\delta_{p}}$ of the linear model are calculated for the height and the speed of refilling.

At the overtaking stage, the tanker's speed is greater than or equal to the speed of the refueled aircraft, and, starting from a distance of $100 \mathrm{~m}$, the preset speed of the tanker aircraft is formed according to the exponential law of an approach

$$
\delta_{\mathrm{ECL}}=K_{V}\left(V_{\mathrm{pc}}-V\right) ; V_{\mathrm{pc}}=V_{\mathrm{rf}}+V_{\Delta D} ; V_{\Delta D}=\left(\Delta D+D_{\mathrm{as}}\right) / T_{\mathrm{exp}},
$$

The autothrottle of the tanker aircraft forms control of thrust by deviation $\left(V_{\mathrm{pc}}-V\right)$. The preset speed $V_{\mathrm{pc}}$ differs from the speed of refueling $V_{\mathrm{rf}}$ by the value $V_{\Delta D}$. And the value of $V_{\Delta D}$ varies according to the exponential law depending on the distance $\Delta D$ between refueling devices.

As already noted at the time of contact, the approach speed should exceed the speed of the refueled aircraft by $1-2 \mathrm{~m} / \mathrm{s}$. Therefore, in the law of formation $V_{\Delta D}$, we introduce the quantity $D_{\text {as }}$ - the depth of occurrence of the asymptote. The asymptote of the exponent must be located behind the drogue's lock. The time constant of the exponent $T_{\exp }$ in the prediction phase is the main wanted control parameter.

With the help of a similar control law, the control of a tanker aircraft is formed.

Any optimal control problem is based on the search for such a control action that ensures the achievement of the objective 


$$
\lim _{t \rightarrow \infty}\left\|\mathbf{x}(t)-\mathbf{r}_{x}(t)\right\|=0, \quad \lim _{t \rightarrow \infty}\left\|\mathbf{u}(t)-\mathbf{r}_{u}(t)\right\|=0
$$

and returns a minimum of the prescribed functional of quality. Here $\mathbf{r}_{x}(t)$ and $\mathbf{r}_{\mathrm{u}}(t)$ are goal vector functions that determine the desired motion of an object with allowance for constraints $\mathbf{x}(t) \in \mathbf{X} \quad \forall \quad t \in[0, \infty)$.

Setting control $\overline{\mathbf{u}}=\mathbf{u}(\tau)$ as a function of time on the interval of time $\tau \in\left[t, t+T_{p}\right]$ and integrating system (2) on the specified interval with initial conditions $\left.\overline{\mathbf{x}}\right|_{\tau=t}=\mathbf{x}(t)$, we obtain a solution $\overline{\mathbf{x}}(t)=\overline{\mathbf{x}}(\tau, \mathbf{x}(t), \overline{\mathbf{u}}(\tau))$ that is interpreted as a prediction of the behavior of the control object with the prediction horizon $T_{p}$.

Formulating the mathematical problem of choosing the optimal control based on the forecast, we will assume that the goal of the control is to provide some given behavior of the model (2), caused, similarly to (3), by vector functions $\mathbf{r}_{x}(t), \mathbf{r}_{x} \in \mathbf{E}^{n}$ and $\mathbf{r}_{u}(t), \mathbf{r}_{u} \in \mathbf{E}^{m}$.

Optimal control for the predictive model is sought by minimizing the functional of the form

$$
J\left(\mathbf{x}(t), \overline{\mathbf{u}}(\cdot), T_{p}, T_{c}\right) \rightarrow \min _{\overline{\mathbf{u}}(\cdot) \in \Omega_{u}}
$$

As the desired control parameter, the time constant of exponent $T_{\exp }$ is chosen, at which the deviation of the coordinate of the point of contact between the fuel probe and the lock of the drogue (the intersection point of the exponential with the plane passing through the lock of the drogue) is minimized. In this case, a multiple forecast is performed with a gradually decreasing interval $\Delta D$. The optimal control $\overline{\mathbf{u}}^{*}$, (in our case $T_{\text {exp }}^{*}$, found for the predictive model on the segment $\left(D-D_{\mathrm{d}}\right)$, is fed to the real object:

$$
\mathbf{u}\left(D-D_{\mathrm{d}}\right) \equiv \overline{\mathbf{u}}^{*}\left(D-D_{\mathrm{d}}\right)
$$

or by specifying control parameters

$$
T_{\exp }\left(D-D_{\mathrm{d}}\right) \equiv T_{\exp }^{*}\left(D-D_{\mathrm{d}}\right)
$$

Next, the control is determined on the prediction interval $\left[\left(D-\Delta D^{*}\right)-D_{\mathrm{d}}\right]$, which is reduced by $\Delta D^{*}$, etc. As the forecast interval decreases, the control accuracy gradually increases.

This method of optimizing control with predictive uses a forecast with a gradual approach to the horizon. Within the framework of this approach, the control is carried out on the principle of feedback with discrete information on the current state of the object at the moment of measurement $\left[\left(D-\Delta D^{*}\right)-D_{\mathrm{d}}\right]$. In this case, the components of the state vector can be either directly measured, or estimated with the help of devices for monitoring the results of measurements of available variables.
The research of the proposed option for automating the approach of refueling devices were carried out by mathematical modeling using the Simulink program, which is part of the mathematical package Matlab (Fig. 3).

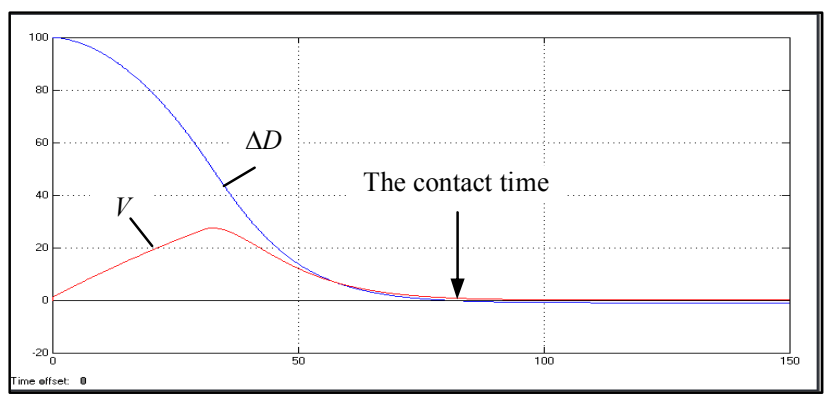

Fig. 3. Results of simulating the process of approach of refueling devices.

The modeling demonstrated the operability of the proposed control algorithm. The time of approach from the initial distance of $100 \mathrm{~m}$ is about 80 seconds. The speed of the tanker aircraft at the contact time can be changed by changing the value of $D_{\text {as. }}$.

The researches were also carried out of the developed docking algorithm for refueling in a turbulent atmosphere. And at the simulation of a turbulent atmosphere was used the generally recognized Dryden model.

Simulation results showed the efficiency of developed algorithms, even when refueling in a turbulent atmosphere, although the approach time in this case is somewhat increased.

After performing the "hitching" in front of the crew of the tanker aircraft that performs the "reverse" AAR, the problem arises of conservation its place in the column. However, this problem is not considered in the article.

Implementation of the proposed automated AAR system will improve safety at the stage of contacting and significantly reduce the time of refueling, which in the manual mode lasts $10-15$ minutes.

\section{CONCLUSIONS}

The technology of "reverse" AAR for regional aircrafts will allow: use these aircrafts at long flights, to increase their commercial payload; significantly save fuel and reduce $\mathrm{CO}^{2}$ emissions into an atmosphere.

The proposed option for automating the refueling device approach using the predictive model, together with the remotecontrolled of drogue will greatly facilitate the piloting technique, will improve safety and accuracy at the contacting stage, reduce the time of refueling and reduces psychophysical loads of pilots.

\section{REFERENCES}

[1] Passenger air service. http://avia.biz.ua/news 23163 -passazhirskie aviaperevozki-2-0.

[2] Project-RECREATE http://www.cruiser-feeder.eu/project

[3] M. K. Filyashkin, "Automation of technology of air-to-air refueling regional aircraft," Electronics and Control Systems, no. 2(48), 2016, pp. 87-91. 